\title{
DIAGNÓSTICO DE ENFERMAGEM E SUA APLICAÇÃO: REVISÃO DA LITERATURA
}

Isabel Cristina Fonseca da Cruz*:

CRUZ, I.C.F. da Diagnóstico de enfermagem e sua aplicação: revisão da literatura. Rev. Esc. Enf. USP, São Paulo, 24(1):149-162, abr. 1990.

Trata-se de um trabalho onde foi realizado um estudo sobre a literatura profissional referente ao Diagnóstico de Enfermagem, a partir da taxonomia proposta pela North American Nursing Diagnoses (NANDA).

UNITERMOS: Diagnóstico de enfermagem. Processo de enfermagem.

\section{INTRODUÇÃO}

A consulta de enfermagem não é uma atividade simples e, assumir o acompanhamento dos problemus de saúde do cliente, requer responsabilidade e preparo por parte do profissional. A utilização de um instrumento que observa as fases do Processo de Enfermagem possibilita a sistematização e, conseqüentemente, a objetividade da consulta.

No entanto, a aplicação do processo, conforme é ressaltado por CRUZ (1988), deve estar associada a um aprofundamento de suas fases, principalmente o histórico e o diagnóstico de enfermagem.

Visando a melhoria da comunicação entre os enfermeiros, têm sido realizados estudos sobre uma taxonomia referente aos diagnósticos de enfermagem aplicados ao cliente, à família e à comunidade.

Nesta parte do estudo foi realizada uma revisão sobre o que existe na literatura profissional referente ao processo e, especialmente, ao diagnóstico de enfermagem, sua conceituação, seu papel dentro do processo de enfermagem e sua aplicação à prática, especialmente junto a clientes que apresentam distúrbios cárdio-vasculares.

\section{Conceitos sobre o Diagnóstico de Enfermagem}

Um diagnóstico é uma conclusão resultante de um processo analítico. A idéia sobre diagnóstico de enfermagem introduz uma responsabilidade quanto a um julgamento clínico entre a coleta de dados e a prescrição dos cuidados.

\footnotetext{
* Enfermeira. Mestre em Enfermagen. Professora Assistente do Departamento de Enfermagem Médico-Cirúrgica da Escola Anna Nery - Universidade Federal do Rio de Janeiro - UFTJ.
} 
HORTA (1972) define desta forma o diagnóstico de enfermagem:

«... é a identificação das necessidades básicas do ser humano que precisam de atendimento e a determinação pela enfermeira do grau de dependência deste atendimento em natureza e extensão».

A autora desenvolve inclusive um modelo operacional para a determinação do grau de dependência, mas observamos que, nesta proposta, a primeira dimensão do diagnóstico de enfermagem ficou sem uma adequação pragmática.

Segundo a avaliação cic CIANCIARULLO (1987), tanto o diagnóstico como o prognóstico apresentam na teoria de Wanda Horta uma construção complexa, exigindo a inclusão destes modelos na prática para a sua validação.

A seguinte definição para diagnóstico de enfermagem é proposta por GORDON (1982):

«O diagnóstico de enfermagem, ou o diagnóstico clínico teito por enfermeiros profissionais, descreve problemas de saúde reais ou potenciais, os quais os enfermeiros devido a sua formação e experiência são capazes e licenciados para tratar».

Em seu estudo sobre o referencial teórico para a classificação de diagnósticos, ROY (1982) apresenta a seguinte conceituação sobre o diagnóstico de enfermagem, resultante de uma discussão no grupo de teoristas de enfermagem, reunidos numa Conferência Bienal da NANDA: "Uma frase suscinta ou um termo que sintetiza um conjunto de indicadores empíricos a respeito dos padrões do homem unitário".

O grupo de estudiosos de enfermagem, atendendo a uma solicitação da NANDA, estabeleceu um referencial teórico para a classificação dos diagnósticos de enfermagem baseado no conceito de homem unitário. Este homem, objeto de estudo da Enfermagem, é um sistema negentrópico, caracterizado por uma organização e por padrões de comportamento.

Os nove padrōes propostos, quando em equilíbrio, manifestam a Saúde. São eles: troca, comunicação, relação, valoração, decisão, atividade, percepşão, conhecimento e sentimento ROY (1984).

$O$ diagnóstico de enfermagem é referenciado desta forma por JONES (1982):

«... é um termo sobre a resposta de uma pessoa a uma situação ou doença que é real ou potencialmente insalubre, para a qual as acóes de enfermagem podem ajudar a mudar em direçáo à saúdex.

KIM et alii (1982) consideraram para os propósitos de seu estudo o diagnóstico como "um termo sobre um problema derivado do histórico que exige ações e objetivos específicos". SCHOEMAKER (1984), realizou uma pesquisa exploratćria, visando esclarecer o significado do termo diagnóstico de enfermagem, tendo chegado a esta definição: 
«O diagnóstico de enfermagem é um julgamento clínico sobre um indfviduo, familia ou comunidade que origina-se de um processo deliberado e sistemático para coleta de dados e análise. O diagnóstico proporciona uma base para as prescrições de terapias pelas quais o enfermeiro é responsável. O diagnóstico é expresso de uma forma concisa e inclui a etiologia quando ela é conhecidà.

Para GORDON (1987), a importância do diagnóstico de enfermagem está no significado que ele dá às observaçóes clínicas. Para esta autora, ele compreende um processo que envolve quatro atividades: a coleta de dados, a interpretação dos dados, a categorização dos dados e a nomeação das categorias.

\section{A relação entre o Diagnóstico e o Processo de Enfermagem}

HORTA (1972) considera fundamental o histórico de enfermagem para a coleta de dados indispensáveis ao diagnóstico. Uma vez coletados, os dados devem ser analisados para a identificação dos problemas de enfermagem..

Embora no Brasil seja adotado, pela maioria dos enfermeiros, o modelo teórico de Horta nas atividades assistenciais, constatamos, através das leituras, que a implementação da teoria não observa a co-relação histórico-diagnóstico enfatizado como fundamental pela teorista.

O diagnóstico de enfermagem é a segunda etapa do processo, porém ainda não o integra totalmente segundo alguns autores (CAMPEDEILLI \& GAIDZINSKY, 1987; MARIA et alii, 1987; CRUZ et alii, 1987).

CAMPEDELLI \& GAIDZINSKY (1987), em seu estudo por exemplo, verificaram que para elaborar a prescrição e a evolução de enfermagem as enfermeiras valiam-se das anotaçōes, evoluções e prescrições de enfermagem anteriores. Também faziam uso de prescrições e evoluções médicas, exames, resultados das discussões e das visitas mé. dicas e, por último, do histórico de enfermagem.

Neste caso, somos levados a refletir sobre o valor das informações obtidas para a análise que precede todo o processo de diagnóstico. Não nos referimos apenas ao diagnóstico como um termo comum, mas ao diagnóstico sobre a situação de saúde do cliente que está a exigir uma conduta de enfermagem adequada e de qualidade.

Por vezes, quando se implementa o processo, as suas fases recebem outros nomes, vemos o diagnóstico ser compreendido como problemas do paciente, ou então as fases são embutidas, desta feita o diagnóstico é parte do histórico.

MARIA et alii (1987) tratando sobre a sistematização da assistência definiram como padrões mínimos: o histórico (levantamento de dados significativos para a enfermagem e identificação de problemas), plano assistencial, evolução e anotação de enfermagem.

CRUZ et alli (1987) relatam que "a partir do histórico de enfermagem, dos registros já existentes e da evolução de enfermagem dos pacientes, são listados em impresso próprio os problemas identificados". 
Este tipo de atendimento dá uma dimensão aos problemas do paciente que, talvez, os diagnósticos de enfermagem não podem e não devem alcançar. Os problemas do paciente geralmente a Enfermagem sozinha não consegue resolver. Justamente por isso o diagnóstico é dirigido exclusivamente para aqueles que têm. solução no âmbito da competência do enfermeiro.

Para CAMPEDELLI \& GAIDZINSKY (1987) o processo de enfermagem não está sendo aplicado em toda a sua plenitude. Segundo estas autoras, apenas o histórico, a evolução e a prescrição de enfermagem são implementados no seu dia a dia.

Mas isto não significa que não se tente implementar a metodologia do processo, incluindo o diagnóstico de enfermagem. Há relatos de experiências neste sentido que nos permitem observar a relação entre as etapas.

De acordo com a visão de MORITZ (1982) o diagnóstico se insere desta forma no processo.

«O processo de enfermagem tem sido definido como uma espiral expansiva de idéias e ação. E evidente que estas idéias e açőes são dirigidas inicial e continuamente para a origem e manutenção dos diagnósticos de enfermagem. Pela inclusão dos fatores etiológicos, dos sinais e sintomas e pelo estabelecimento dos objetivos nos quais o plano é baseado, as açóes de enfermagem passam a tratar os diagnósticos de enfermagem. A evoluçào, uma etapa implícita em todo o processo, não é uma atividade de julgamento, mas um método de refinamento da prescrição».

$\mathrm{Na}$ avaliação de MILLER et alii (1987):

«O diagnóstico de enfermagem, como um componente importante do Processo, proporciona uma taxonomia comum para descrever os problemas que os enfermeiros podem tratar e é um modo conveniente e lógico para referenciar os padrões de enfermagem».

Segundo SPECHT \& DREY (1987), a aplicação dos Diagnósticos de Enfermagem tornou a identificação dos problemas mais acurada e ajudou no planejamento da assistência. Enfim, aprimorou as habilidades necessárias para a implementação do Processo.

Os estudos sobre a taxonomia dos diagnósticos de enfermagem pretendem criar uma linguagem universal entre os enfermeiros referente aos problemas de saúde de seus clientes. E uma tarefa árdua! Principalmente quando verificamos a prolixidade de termos para tratar as mesmas coisas. Vimos que problemas do paciente pode, conforme o contexto, significar diagnóstico de enfermagem. Pelas leituras feitas, verificamos agora uma insistência na expressão "problema de enfermagem" como substituição para diagnóstico.

Vários autores entendem problema de enfermagem como sinônimo de diagnóstico (SANTOS et alii, 1987; CAR, 1987).

Por exemplo, MARIA et alii (1987) introduzem o Plano Assistencial, visando a resolução ỏos problemas de enfermagem identificados. 
Segundo HORTA (1979), após a análise dos dados colhidos são identificados os problemas de enfermagem. Dai então, em nova análise, buscam-se as necessidades básicas afetadas e o grau de dependência do paciente.

FRIEDLANDER (1977) percebeu a existência de uma confusão na terminologia utilizada pelos autores quando se referiam a "problemas de enfermagem".

$\mathrm{Na}$ sua opinião, são raros os problemas de enfermagem e explica desta forma:

\begin{abstract}
«O que existe são problemas do paciente em geral, tão complexos que sua solução só é passivel mediante a atuação de vários profissionais. Nấ sగ a marcante especialização dos profissionais, como a variedade de aspectos oferecidos por um só problema, faz nos acreditar que cada problema do paciente tem apenas alguns aspectos nos quais a enfermagem atua. Nesse caso não o podemos denominar problema de enfermagem». (Friedlander, 1977).
\end{abstract}

ANGERAMI \& CARVALHO (1987) concluem que as divergências quanto a terminologia podem constituir uma limitação para a aplicação prática do processo ou para o próprio desenvolvimento do conhecimento. Verificaram também que "diagnóstico" e "avaliação" são entendidos como iguais. Sobre os diferentes termos utilizados quanto ao processo de enfermagem manifestaram que:

«O proṕsito do estudo meste campo é o de afastar homônimos para se obter uma definição inequívoca de significado. Portanto a problemática relaciona-se em determinar o significado do signo e que convém ao signo».

Neste sentido, FRIEDLANDER (1977) também mostra sua crença na luta dos enfermeiros para universalizar a linguagem, construir uma terminologia própria, efetivando assim a comunicação.

\title{
Desenvolvimento histórico
}

SLMÓES (1980) diz ser indispensável para o desenvolvimento da profissão a existência de uma terminologia. Os termos empregados por um grupo profissional devem transmitir a todos o mesmo significado.

Contudo, a preocupação de SIMŌES (1980) difere da nossa na medida em que desejamos melhorar a qualidade da comunicação da enfermagem sobre os problemas de saúde que afetam o nosso cliente.

BARBATO et alii (1979) relatam uma experiência onde tiveram a necessidade de levantar os problemas dos clientes mais freqüentes, a fim de mais facilmente orientar os profissionais de enfermagem que ingressavam na unidade coronariana.

BHERING et alii (1971) apresentam a sua experiência de implantação do Diagnóstico de Enfermagem, de acordo com a Teoria de Wanda Horta. Terminaram por concluir que tanto as necessidades básicas, como o grau de dependência são de difícil definição e mensuração. 
HORTA (1977) observou que na aplicação do processo de enfermagem o enfermeiro encontra uma grande dificuldade para estabelecer o diagnóstico. Atribuiu como causas: o desconhecimento de sintomas (problemas de enfermagem), de necessidades básicas alteradas e da nomenclatura destas necessidades, entre outras.

Baseadas na teoria de Carkuff, LEITE et alii (1981) descrevem a sua experiência em identificar os problemas dos pacientes e traçar ações de enfermagem específicas para resolvê-los.

Estas experiências, pelo menos no Brasil, segundo o nosso entendimento, não conseguiram somar esforços que levassem ao desenvolvimento de uma terminologia comum para a profissão.

Na década de setenta, foi criada na América do Norte uma associação, a NANDA, que coordena as pesquisas nesta área. Estes estudos culminaram recentemente na criação de uma taxonomia. É GORDON (1987) que diz:

\begin{abstract}
«Em 1986, a North American Nursing Diagnoses Association (NANDA) publicou a Taxonomia I. Este sistema de classificação contém todos os diagnósticos que submeteram-se a uma revisão. A classificação é organizada em nove padróes humanos de comportamento identificados pelas teoristas de enfermagem durante o seu trabalho na Associaçáa, no periodo de 1977 a 1982. O aprimoramento da Taxonomia I continuará sendo objeto de discussão nas próximas conferências bienais».
\end{abstract}

Todavia, a Taxonomia proposta pela NANDA, em 1986, não é a única que está sendo submetida à apreciação e à validação pelos enfermeiros.

WEIDMANN \& NORTH (1987) utilizam o sistema de classificação de Omaha. Este sistema foi desenvolvido pela Associação de Enfermeiras Visitadoras, da cidade de Omaha, estado de Nebraska.

O sistema de Omaha lista os problemas dos clientes relativos à área de Saúde Comunitária. No entanto as autoras acreditam que no futuro as duas classificações comporão uma só, atendendo assim a todos os campos da enfermagem.

\title{
$A$ estrutura do diagnóstico
}

De acordo com GORDON (1987) as ações de enfermagem são parte de uma aprendizagem prévia sobre diagnóstico-tratamento-objetivo ligados entre si e guarảacos na memória, sendo que o diagnóstico possui três componentes básicos, a saber:

a) o problema que refere-se ao estado do cliente, expresso de uma forma concisa;

b) os fatores etiolćgicos que relacionam-se com as causas do problema de saúde do cliente;

c) e os sinais e os sintomas que permitem uma melhor discriminação entre os problemas de saúde. 


\section{A aplicação do diagnóstico de enfermagem}

Ao observar pacientes com desordens cárdio-vasculares, KIM et alii (1982) identificaram e validaram 12 diagnósticos de enfermagem, tais como: diminuição do débito cardíaco, alteração na circulação coronária, ansiedade, déficit de conhecimento e altereção na imagem corporal, entre outros.

RANTZ \& MILLER (1987) notaram que após a implementação da Taxonomia, em seu Serviço, aconteceu uma melhora na comunicação interdisciplinar e no planejamento da assistência, sendo enfocada as necessidades individuais dos clientes.

Para McCOURT (1987) a implementação dos Diagnósticos de Enfermagem deve ser acompanhada de uma orientação sobre as intervenções mais apropriadas, visando não só guiar o cuidado, mas também reduzir o número de erros.

Referindo-se a Consulta de Enfermagem, NOGUEIRA (1980) considera que o Diagnóstico deve ter um rendimento grande, caso contrário perde de vista o objetivo de aumentar a cobertura. Por isso, em seu estudo, o diagnóstico de enfermagem é constituído de uma rápida avaliação da dependência, em relação aos itens que representam as necessidades do cliente.

CRUZ (1988) testou um instrumento na Consulta de Enfermagem ao cliente hipertenso onde foram categorizados 15 diagnósticos de enfermagem, estando alguns de acordo com a terminologia proposta pela NANDA, tais como: déficit de conhecimento, inaderência, ansiedade e relacionamento familiar ineficaz. A autora ressalta a dificuldade para se estabelecer um diagnóstico de enfermagem e sugere que sejam realizados estudos para a formação de uma taxonomia de enfermagem.

Referindo-se a consulta de enfermagem ao binômio mãe-filho, CAMPEDELLI (1986) observou que embora os enfermeiros utilizassem um vocabulário diversificado, os problemas dos clientes não variavam. A autora alerta inclusive para a necessidade de se definir um vocabulário próprio.

A importância de uma taxonomia de enfermagem não se restringe às atividades assistenciais, segundo GORDON (1987), o seu valor estende-se igualmente para o ensino de enfermagem e para as pesquisas. $O$ desenvolvimento de uma terminologia própria da enfermagem traz implícito o desenvolvimento do seu corpo de conhecimentos.

\section{Considerações finais}

Pelos conceitos encontrados na literatura consultada, vemos que, no Brasil principalmente, são poucos os estudos dirigidos para a criação de uma taxonomia de enfermagem ou para a validação das propostas existentes (vide em anexo a Taxonomia I).

A importância de uma classificação sobre diagnóstico de enfermagem para o desenvolver o corpo de conhecimentos da profissão e, con- 
seqüentemente, dar-lhe um perfil mais nitido, somada a escassez de literatura nacional pertinente, denuncia a premência de estudos sobre o tema em pauta.

CRUZ, I.C.F. da Nursing diagnosis and its aplication: review of the literature. Rev. Esc. Enf. USP, São Paulo, 24(1):149-162, Apr. 1990.

This paper refers to a study on the profissional bibliografy related to the Nursing Diagnosis as the propposed by North American Nursing Diagnosis Association (NANDA).

UNITERMS: Nursing diagnosis. Nursing processo.

\section{REFERENCIAS BIBLIOGRÁFICAS}

1. ANGERAMI, E.L.S. \& CARVALHO, E.C.D.E. Processo de enfermagem: reflexões acerca da terminologia utilizada. Rov. Ksec. Enf. USP, São Paulo, 21 (nº esp.):29-33,jun. 1987.

2. BARBATO, M.G. et alii. Principais problemas físiológicos apresentados por pacientes com infarto do miocárdio e as respectivas prescrições rle enfermagem. Rev. Esc. Enf. USP, Sãı) Paulo, 13(2):147-55, ago. 19779.

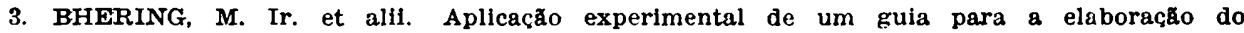
diagnóstico de enfermagem no Hospital São Paulo. Rev. Bras. Enf., Rio de Janeiro, 24(5):89-102, jul./set. 1971.

4. CAMPEDELLI, M.C. \& GAIDZINSKY, R.R. A metodolo isia assistencial de enfermagem na prática: hospital universitário da USP. Rev. Esc. Enf. USP, Săo Paulo, 21 (no esp.): :62-7, jun. ?1987.

5. CAMPEDELLI, M.C. A Consulta de enfermagem ao dinômio mãe c filho: análise das condutas de enfermagem. São Paulo, 1986. 118p. ('Tese de doutorado - Faculdade de Saúde Pública/USP).

6. CAR, M.R. Identificação de problemas de enfermagem la esfera rísica sm unidade de terapia intensiva. Rov. Esc. En . USP, Săo Paulo, 21(1):23-36, abr. 1987.

7. CIANCIARULLO, T.I. Teoria das necessidades humanas bisicas: um marco indelével na enfermagem brasileira. Rev. Esc. Enf. USP, São Paulo, 21 (n esp.):100, jun. 1987.

8. CRUZ, D. de A.L.M. da et alii. Sistematização da assistência de eutermagem cm uma área do recuperação da saúde. Rev. Esc. Fñ. USP, Sã̃ Paulo, 21 (ne esp.):68-76, jun. 1987.

9. CRUZ, I.C.F. da. Consulta de enfermagem ao cliente hipertenso. Rio de Janciro, UFRJ, 1988.

10. FRIEDLANDER, M.R. Problemas de enfermagem e sua crinceituaçãu. Rev. Esc. Enf. USP, São Paulo, 11(3):325-30, dez. 1977 .

11. GORDON, M. Frameworks for the diagnostic process. In:-—_. Nursing diagnoses: process and application. New York, Mc-Graw-Hill, 1982. Cap. 3. p.63-109.

12. - Implementation of nursing diagnoses: an overview. Nurs. Clin. North Am., Philadelphia, 22(4):875-9, Dec. 1987.

13. HORTA, W A. Histórico. In:——. Processo de mnfermagem. São Faulo, EPU, 1979. Cap. 2. p.37-8.

14. - Diagnóstico de enfermagem: representação gráfiç. Fnf. Novas Mimens., São Paulo, :(2):75-7, mar./abr. 1977. 
15. Diagnóstico de enfermagem: estudo básico da determinação da dependência de enfermagem. Rov. Bras. Enf., Rio de Janciro, 25(1):267-73, jul./set. 1972.

16. JONES, P.E. The revision of nursing diagnoses terms. In: NATIONAL CONFERENCES CLASSIFICATION OR NURSING DIAGNOSES, 3., NeW York, 1982. I'roceedings, New York, MCGraw-Hill, 1982.

17. KIM, M.J. et alii. Clinical use of nursing diagnoses in cardiovascular nursing. In: NATIONAL CONFERENCE CLASSIFICATION OF NURSING DIAGNOSES, 3., New York, 1982. Proceedings, New York, McGraw-Hill, 1982.

18. LEITE, T.L. et alli. A relação de ajuda a um grupo cé clientes com problemas de locomoção. Bov. Bras. Enf., Brasilia, 34(3/4):211-23, jul./dez. 1981.

19. MARIA, V.L.R. et alii. Sistemaização da assistência dı enfermagem no Instituto «Dante F'azżnese» de cardiologia: relato de experiéncia. Rov. Fac. Ent. USP, Săo Paulo, 21 (no esp):77-87, jun. 1987.

20. McCOURT, A.E. Implementation of nursing diagnoses through integration with quality assurance. Nurs. Clin. North Am. Phlladelphia, 22(4):889-904, Dec. 1997.

21. MLLER, J. et alii. The impact of nursing disgnoses in a longterm care setting. Nurs. Clin. North Am., Philadelphia, 22(4):905-1》, Dec. 1987.

22. MORITZ, D.A. Nursing diagnosis in relation to the nursing proceas. In: NATIONAL CONFERENCE CLASSIIICATION OF NURSI:TG DIAGNOSIS, 3. New York, 1982. Proceedings, Ncw York, MCGrcw-Hill, 1982.

23. NOGUEiRA, M.J. de C. Consulta de enfermegem em saride da criança: modelo operacional. Rev. Esc. Ene. USP, Săo Paulo, 14(1):29-49, abr. 1980.

24. RANTZ, M.J. \& MILLFR, T.V. Change theory: a framework for implementing nursing diagnoses in a long-term care setting. Nurs. Clin. North Am., Philadelphia, 22(4): 887-97, Dec. 1987.

25. ROY, S.C. Historical perspective of the theoretical framework for thc classification on nursing diagnosis. In: NATIONAL CONFERENCE CLASSIFICATION OF NURSING DIAGNOSIS, 3., New York, 1982. Procedings, New York, McGraw-Hill, 1982.

26. ROY, S.C. Framework for classification systems development: progress and issues. In: NATIONAL CONFERENCE CLASSIFICATION OF NURSING DLAGNOSES, 5., Saint Louis, 1984. Proceodings, St. Louis, Mosby, 1984.

27. SANTOS, L.C.R. et alil. O ensino da metodologia da assistencia de enfermagem: responsabilidude da disciplina de fundamentos de enfermagem? Rev. Esc. Enf. USP, Săo Paulo, 21(1):75-88, abr. 1987.

28. SCHOEMIAKER, T.K. Essential features of a nursing diagnoses. In: NATIONAL CONFERENCE CLASSIFICATION OF NURSING DIAGNOSIS, 5., St. Louis, 1984. Proceedings, St. Luis, Mosby, 1984.

29. SIMOES, C. Ir. Contribuiç̆̊ a estudo da terminologia básiç de enfermagem no Brasil: taxonomir e conceituaçăo. Bauru, 1980. 185p. (Tese de niestrado - Escola de Enfermagem Ana Nert - UFRJ).

30. SPECHT, J. \& DREY, K. The implementation o nursing diagnoses: the Iowa Veteran Home experience. Nurs. Clin. North Am., Philadelphia, 22(4)::917-33, Dec. 1987. 


\section{ANEXO I}

TAXONOMIA DOS DIAGNOSTTCOS DE ENFERMAGEM I *

Proposta da North American Nursing Diagnoses Association (1986)

1 - Troca: padrâo de comportamento humano que envolve o processo mútuo de captação e eliminação (dar e o receber).

1.1 - Alteraçoes na nutrição

1.1.1 - (celular)

1.1 .2 - (sistêmica)

1.1.2.1 - mais do que as necessidades corporais;

1.1.2.2 - menos do que as necessidade corporais;

1.1 .23 - potencial para mais do que as necessidades corporais.

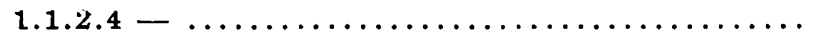

1.2 - Alteração na regulação física

1.2.1 - (resposta imune)

1.2.1.1 - potencial para infeç̧̃o

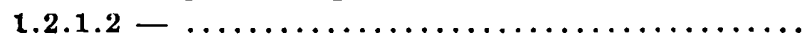

1.2.2 - alteração na temperatura corporal

1.2.2.1 - potencial

1.2.2.2 - hipotermia

1.2.2.3 - hipertemia

1.2.2.4 - termo-regulação ineficaz

1.2.2.5 -

1.3 - Alteração na eliminação

1.3.1 - via intestinal

1.3.1.1 - constipação

1.3.1.2 - diarréia

1.3.1.3 - incontinência

1.3.2 - via urinária

1.3.2.1 - incontinéncia

1.3.2.1.1 - estresse

1.3.2.1.2 - reflexa

1.3.2.1.3 - urgéncia

1.3.2.1.4 - funcional

1.3.2.1.5 - total

1.3 .2 .2 - retenção

1.3 .3 - (pele)

$1.3 .3 .1-\ldots \ldots \ldots \ldots \ldots \ldots \ldots \ldots \ldots \ldots \ldots \ldots \ldots$

1.3.3.1 - $\ldots \ldots \ldots \ldots \ldots \ldots \ldots \ldots \ldots \ldots \ldots \ldots \ldots \ldots$

1.3.3.2 - $\ldots \ldots \ldots \ldots \ldots \ldots \ldots \ldots \ldots \ldots \ldots \ldots \ldots \ldots \ldots$

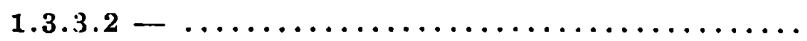

1.4 - Alteração na circulação

1.4.1 - (vascular)

- As categorias entre chaves indicam aquelas que não foram tormalmente revistas e votadas. Os espaços em branco denotam os diagnósticos que ainda rıão foram descrixos. 
1.4.1.1 - perfusão tecidual

1.4.1.1.1 - renal

1.4.1.12 - cerebral

1.4.1.1.3 - cárdio-pulmonar

1.4.1.1.4 - gastro-intestinal

1.4.1.1.5 - periférica

1.4.1.2 - volemia

1.4.1.2.1 - excesso

1.4.1.2.2 - déficit

1.4.1.2.2.1 - renal

1.4.1.2.2.2 - potencial

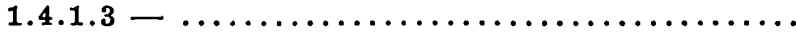

1.4 .2 - (cardiaca)

1.4.2.1 - diminuição do débito cardíaco

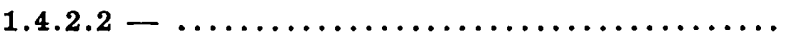

1.5 - Alteraçðes na oxigenação

1.5.1 - (respiração)

1.5.1.1 - diminuição da troca gasosa

1.5.1.2 - limpeza ineficaz das' vias aéreas

1.5.1.3 - padrão respiratório ineficaz

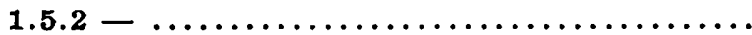

1.6 - Alteração na integridade física

1.6.1 - potencial para injúria

1.6.1.1 - potencial para sufocamento

1.6.1.2 - potencial para envenenamento

1.6.1.3 - potencial para trauma

1.6.2 - enfraquecimento (diminuição)

1.6.2.1 - integridade da pe'e

1.6.2.1.1 - real

1.6.2.1.2 - potencial

1.6.2.2 - integridade do tecido

1.6.2.2.1 - mucosa oral

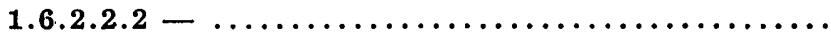

1.6.2.2.3

1.6 .2 .3

2 - Comunicação: padrão ce comportamento humano referente ao intercambio de mensagens.

2.1 - Alteração na comunicação

2.1.1 - verbal

2.1.1.1 - diminuiç̃̃o

2.1.1.2 - $\ldots \ldots \ldots \ldots \ldots \ldots \ldots \ldots \ldots \ldots \ldots \ldots \ldots \ldots \ldots$

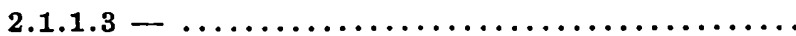

2.1 .2 - (não verbal)

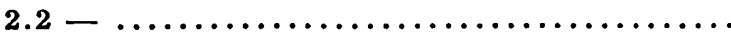

2.3

2.3 .1 
3 - Relação: padrão de comportarnento que denota o estabelecimento de ligações:

3.1 - Alterações na socialização

3.1.1 - interasãa social reduzida

3.1 .2 - isolamento social

$3.1 .3-$

3.2 - Alteraçoes na função social

3.2.1 - (desempenho da função)

3.2.1.1 - paternidade/maternidade

3.2.1.1.1 -- real

3.2.1.1.2 - potencial

3.2.1.2 - sexual

3.2.1.2.1 - disfunção

3.2 .1 .2 .2

3.2 .1 .2 .3

3.2.1.3 - profissional

3.2.2 - processo familiar

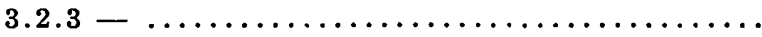

3.3 - Alteração no padrão de sexunlidade

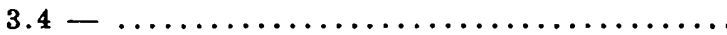

4 - Valoração: paḍão de comportamento que exprime os valores relativos.

4.1 - Alteração no estado espiritual

4.1.1 - angústia (distress)

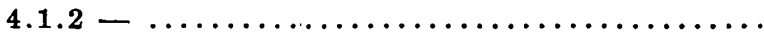

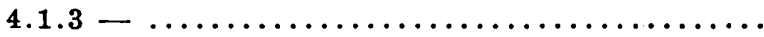

$4.2-\ldots \ldots \ldots \ldots \ldots \ldots \ldots \ldots \ldots \ldots \ldots \ldots \ldots \ldots \ldots$

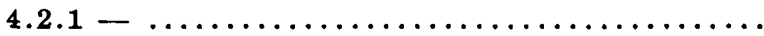

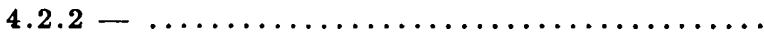

5 - Decisão: padrão de comportamento humano referente a seleção de alternativas

6.1 - Alteração no processo de adaptạ̧̃o

5.1.1 - individual

5.1.1.1 - ineficaz

5.1.1.1.1 - diminưgão no ajustamento

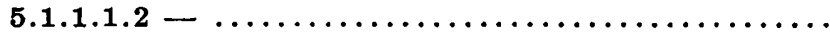

5.1.1.2

5.1 .2 - familier

5.1.2.1 - incificaz

5.1.2.1.1 - desestinulante

5.1.2.1.2 - aliciante

5.1.2.2 - patencial para crescimento/desenvolvimento

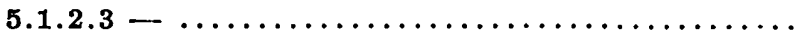

5.1 .3 - (comunitário)

5.2 - Alteraç⿰丿㇄ na participação

5.2 .1 - (individual)

5.2.1.1 - inaderência

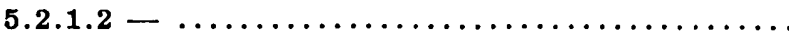


5.2 .1 .3

5.2 .2 - (familiar)

5.2.3 - (comunitária)

6 - Atividade: padrão de comportamento ligado às ações e movimentos.

6.1 - Alterações na atividade

6.1.1 - mobilicade física

6.1.1.1 - diminuída

6.1.1.2 - intolorancia a ativiáade

6.1.1.3 - potencial de intolerância a atividade

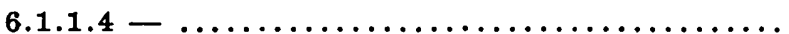

6.1.2 - (mobilidace social)

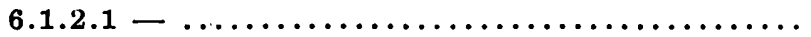

6.1.2.2 -

6.2 - Alteração no repouso

6.2.1 -- distúrbio no padrão de sono

$6.2 .2-\ldots \ldots \ldots \ldots \ldots \ldots \ldots \ldots \ldots \ldots \ldots \ldots \ldots \ldots$

6.3 - Alteraçős no lezer

6.3.1 - atividades diversionais

6.3.1.1 - déficit

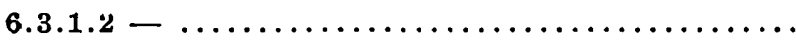

6.3 .2

6.4 - Alterações nas atividades diárias

6.4.1 - gerenciamento da manutenção do lar

6.4.1.1 - diminuição

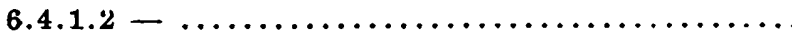

6.4.2 - manutenção da saúde

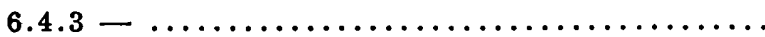

6.5 - Alterações no auto-clidado

6.5.1 - alimextaçấ

6.5.1.1 - diminuição da deglutiçăo

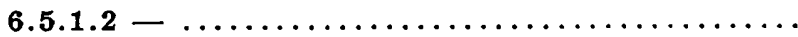

6.5.1.3

6.5.2 - banho/higiene

6.5.3 - vestir-se/calçar-se

6.5.4 - toilette

6.6 - Alteração no crescimento e desenvolvimento

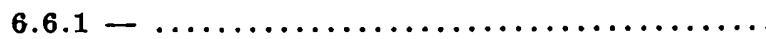

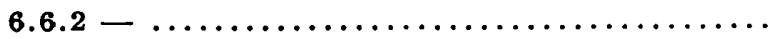

7 - Percepção: padrão de comportamento humano que envolve a recepçåo de informaço

7.1 - Alteraçoses no auto-conceito

7.1.1 - distúrbio na imagem corporal

7.1.2 - distárbio na auto-estima

7.1.3 - distúrbio na identidade pessoal

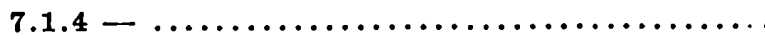

7.2 - Alteração na percepç̧ão/sensibilidade

7.2.1 - visual 
7.2.1.1 - unilateral

7.2.1.2

7.2.2 - auditiva

7.2 .3 - cinestésica

7.2.4 - gustativa

7.2.5 - olfativa

7.3 - Alteraçóes no sentido da vida

7.3.1 - desesperança

7.3 .2 - impoténcia

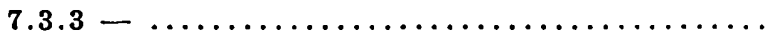

8 - Conhecimento: padrão humano de comportamento relativo ao conteúdo associado a mensagem.

8.1 - Alterações no conhecimento

8.1.1 - déficit

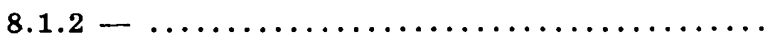

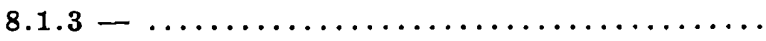

8.2 - Alterações na aprendizagem

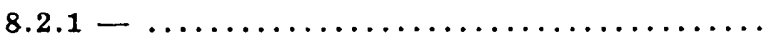

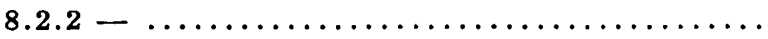

8.3 - Alterações no processo de pensamento

8.3.1 - (confusão)

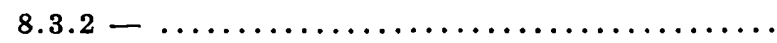

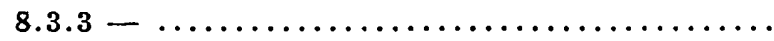

9 - Sentimento: padrão de comportamento relacionado a compreensão subjetiva de una informação.

9.1 - Alteraçoes no conforto

0.1.1 -dor

9.1.1.1 - crônica

9.1.1.2 - (aguda)

9.1.1.3 - ........

9.2 - Alteraçóes na integridade emocional

9.2.1 - ansiedade

9.2.2 - luto

9.2.2.1 - existencial

9.2.2.2 - antecipado

9.2 .2 .3

9.2.3 - potencial para violência

9.2.4 - medo

9.2.5 - resposta pos-trauma

9.2.5.1 - sindrome do estupro

9.2.5.1.1 - estupro (trauma)

9.2.5.1.2 - «compound reaction»

925.1 .3 - reação silenciosa

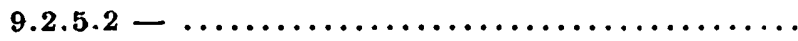

9.2 .6

9.2 .7

9.3 